\title{
What Do We See in Spectra?: Assignment of High-Intensity Peaks of Cutibacterium and Staphylococcus Spectra of MALDI-TOF Mass Spectrometry by Interspecies Comparative Proteogenomics
}

\author{
Itaru Dekio ${ }^{1, *,+}\left(\mathbb{D}\right.$, Yuki Sugiura ${ }^{1}$, Susumu Hamada-Tsutsumi ${ }^{2}$, Yoshiyuki Murakami ${ }^{3}$, Hiroto Tamura ${ }^{2}$ \\ and Makoto Suematsu ${ }^{1}$ \\ 1 Department of Biochemistry \& Integrative Medical Biology, School of Medicine, Keio University, \\ 35 Shinanomachi, Shinjuku-ku, Tokyo 160-8582, Japan; yuki.sgi@gmail.com (Y.S.); gasbiology@keio.jp (M.S.) \\ 2 Department of Environmental Bioscience, Meijo University, 1-501 Shiogamaguchi, Tenpaku-ku, Nagoya, \\ Aichi 468-8502, Japan; tsusumu07@gmail.com (S.H.-T.); hiroto@meijo-u.ac.jp (H.T.) \\ 3 Seikakai Mildix Skin Clinic, 3rd Floor, 3-98 Senju, Adachi-ku, Tokyo 120-0034, Japan; bow.t@aol.jp \\ * Correspondence: i@skinmicrobe.com; Tel.: +81-3-3433-1111 (ext. 3341); Fax: +81-3-5401-0125 \\ + Present Address: Department of Dermatology, The Jikei University School of Medicine, \\ 3-25-8 Nishi-shinbashi, Minato-ku, Tokyo 105-8461, Japan.
}

Citation: Dekio, I.; Sugiura, Y.; Hamada-Tsutsumi, S.; Murakami, Y.; Tamura, H.; Suematsu, M. What Do We See in Spectra?: Assignment of High-Intensity Peaks of Cutibacterium and Staphylococcus Spectra of MALDI-TOF Mass Spectrometry by Interspecies Comparative Proteogenomics. Microorganisms 2021, 9, 1243. https://doi.org/10.3390/ microorganisms 9061243

Academic Editor: Adriana Calderaro

Received: 28 April 2021

Accepted: 3 June 2021

Published: 8 June 2021

Publisher's Note: MDPI stays neutral with regard to jurisdictional claims in published maps and institutional affiliations.

Copyright: (c) 2021 by the authors Licensee MDPI, Basel, Switzerland. This article is an open access article distributed under the terms and conditions of the Creative Commons Attribution (CC BY) license (https:// creativecommons.org/licenses/by/ $4.0 /)$.

\begin{abstract}
Matrix-assisted laser-desorption/ionization time-of-flight (MALDI-TOF) mass spectrometry is a widely used and reliable technology to identify microbial species and subspecies. The current methodology is based on spectral fingerprinting, analyzing protein peaks, most of which are yet to be characterized. In order to deepen the understanding of these peaks and to develop a more reasonable identification workflow, we applied proteogenomic approaches to assign the high-intensity peaks of MALDI-TOF spectra of two bacterial genera. First, the 3-22 kD proteomes of 5 Cutibacterium strains were profiled by UPLC-MS/MS, and the amino acid sequences were refined by referring to their genome in the public database. Then, the sequences were converted to $\mathrm{m} / \mathrm{z}$ ( $x$-axis) values based on their molecular masses. When the interspecies comparison of calculated $\mathrm{m} / \mathrm{z}$ values was well-fitted to the observed peaks, the peak assignments for the five Cutibacterium species were confirmed. Second, the peak assignments for six Staphylococcus species were performed by using the above result for Cutibacterium and referring to ribosomal subunit proteins coded on the S10-spc-alpha operon (the S10-GERMS method), a previous proteomics report by Becher et al., and comprehensive genome analysis. We successfully assigned 13 out of 15 peaks for the Cutibacterium species and 11 out of 13 peaks for the Staphylococcus species. DNA-binding protein HU, the CsbD-like protein, and 50S ribosomal protein L7/L12 were observed in common. The commonality suggests they consist of high-intensity peaks in the MALDI spectra of other bacterial species. Our workflow may lead to the development of a more accurate species identification database of MALDI-TOF mass spectrometry based on genome data.
\end{abstract}

Keywords: Cutibacterium; Staphylococcus; proteomics; MALDI-TOF mass spectrometry; high-intensity peaks; S10-GERMS

\section{Introduction}

Matrix-assisted laser desorption/ionization time-of-flight (MALDI-TOF) mass spectrometry is a widely used technology to identify microbial isolates in clinical settings. The smear of a bacterial isolate on a metal plate is processed with or without an extraction process, followed by crystallization by application of a matrix solution; then, a LASER desorbs the crystallized matrix and sample to form a cloud of positive ions. By the voltage difference of the target plate and the background, these ions are pulled through a vacuum tube. Ions with smaller mass $(\mathrm{m} / \mathrm{z}$ value) fly faster to reach the detector at the other end 
of the tube, finally giving a spectrum of the ionized molecules. The spectral peak pattern of the 2-20 kD range is of low molecular weight proteins, used for species- or subspecieslevel identification using a manually created database. In some cases of closely related species like Streptococcus, MALDI-TOF can identify bacterial colonies when 16S rRNA gene identification is not successful [1].

Although this fingerprinting method is widely used and considered relatively reliable, misidentification occurs due to inaccuracy of the database derived from the manual collection of spectral data. To improve the identification database, peak calculation should be based on digital analysis based on the genome. However, most of the peaks are unannotated, leaving these spectra as a black box. Theoretically, many of these peaks are believed to be ribosomal subunit proteins [2], and identification protocol using these proteins are being developed (the S10-GERMS method) [3]. However, the peaks of the ribosomal subunits are relatively small, and most of the key prominent peaks for species identification remain unidentified [4]. Therefore, an investigation into nonribosomal proteins to identify these peaks is of utmost importance to develop a next-generation database. Teramoto et al. have identified two of a dozen prominent peaks in the spectra of Cutibacterium acnes that are used in subspecies discrimination-a CsbD-like protein and a $7 \mathrm{kD}$ antitoxin [4] - and this is the first report of this kind in bacterial MALDI-TOF spectra. The postsource decay (PSD) effect in the commonly used setting for MALDI-TOF species identification, which includes linear mode with $\alpha$-cyano-4-hydroxycinnamic acid (HCCA/CHCA) as the matrix, is faint $[5,6]$ and can be ignored as an origin of prominent peaks. Therefore, the ions consisting of such peaks should be proton-added protein molecules without a PSD effect. The report of Teramoto et al. proves that at least a part of the proteins, consisting of the prominent peaks, are such crude proton-added nonribosomal proteins.

The reason why most of the annotations are not yet reported may be due to three reasons. First, the proteins encoded in the genome are not always expressed, so a topdown approach from the genome will have difficulty picking up the actual proteins in the specimen. Second, the bottom-up approach is also problematic. Overall, analysis of the protein included in the specimen using MS/MS is troublesome because the sample includes hundreds of proteins, so the peptide production by trypsin digestion results in more than a thousand peptides that cannot be annotated to the correct proteins. Third, the protein database does not include all proteins in a strain; additionally, the amino acid sequence often lacks parts of the proteins.

Cutibacterium and Staphylococcus are two major bacterial genera residing on human skin. Thus, these are often included in clinical specimens to be sent to hospital laboratories. Genus Cutibacterium, with five species including Cutibacterium acnes (previously, Propionibacterium acnes) [7], was recently coined [8] but is of particular importance as the genus is related to diseases not only of the skin, such as acne vulgaris, but also of internal organs, such as sarcoidosis [9] and prostate cancer [10]. Genus Staphylococcus includes 55 species [7], and the genus is also clinically important in various infections. These genera are both Gram-positive and they are included in different major bacterial phyla, namely, Actinobacteria and Firmicutes. This means the common features of Cutibacterium and Staphylococcus may be applied to many bacterial species.

In this work, we aim to assign the major peaks of MALDI spectra of these genera by comparing the proteome profile and MALDI spectra using a bottom-up approach with precise techniques, including gel excision and the curation of a database-derived amino acid sequence with the whole genome.

\section{Materials and Methods}

\subsection{Selection of Bacterial Strains}

Eight strains of genus Cutibacterium and seven strains of genus Staphylococcus were obtained from Japan Collection of Microorganisms (JCM); National Collection of Type Cultures (NCTC), United Kingdom; Culture Collection of University of Goteborg (CCUG), Sweden; and Monash University, Melbourne, Australia (Table 1). The full genome se- 
quences of these strains are available from the DDBJ/EMBL/GenBank public database. For Cutibacterium species, strains were grown on trypticase soy agar supplemented with $5 \%$ sheep blood (BD, Franklin Lakes, NJ, USA) for 7 days under anaerobic conditions $\left(0 \% \mathrm{O}_{2} / 10 \% \mathrm{CO}_{2}\right)$, created in an anaerobic jar with an AnaeroPack sachet (Mitsubishi Gas Chemical, Tokyo, Japan) at $37^{\circ}$ C. Moreover, for C. acnes subsp. acnes JCM $6425^{\mathrm{T}}$, C. acnes subsp. acnes JCM 18918, and C. acnes subsp. elongatum JCM $18919^{\mathrm{T}}$, cultivation was performed under a normal atmosphere $\left(21 \% \mathrm{O}_{2} / 0.04 \% \mathrm{CO}_{2}\right)$, and different oxygen pressures for culture were established by the hypoxia workstation Invivo 2 (Baker Ruskinn, Bridgend, Wales, UK) for $12 \% \mathrm{O}_{2} / 9 \% \mathrm{CO}_{2}, 6 \% \mathrm{O}_{2} / 15 \% \mathrm{CO}_{2}$ and $2 \% \mathrm{O}_{2} / 19 \% \mathrm{CO}_{2}$. For Staphylococcus species, strains were grown on trypticase soy agar supplemented with $5 \%$ sheep blood (BD) for 2 days under air $\left(21 \% \mathrm{O}_{2} / 0.04 \% \mathrm{CO}_{2}\right)$.

Table 1. Strains used. T after strain number indicates type strains of species/subspecies. * Identical to the used strain (same strain deposited from different culture collections).

\begin{tabular}{|c|c|c|}
\hline Species & Strain Number & Genome Entry \\
\hline \multirow[t]{2}{*}{ Cutibacterium acnes subsp. acnes } & JCM $6425^{\mathrm{T}}$ (Type IA 1 ) & CP044255 (ATCC 6919T *) \\
\hline & JCM 18918 (Type IB) & BAVO01 (JCM 18918) \\
\hline Cutibacterium acnes subsp. defendens & $\mathrm{JCM} 6473^{\mathrm{T}}$ & CP003084 (ATCC $11828^{\mathrm{T} *}$ ) \\
\hline Cutibacterium acnes subsp. elongatum & JCM $18919^{\mathrm{T}}$ & BFFM01 (JCM 18919T) \\
\hline Cutibacterium avidum & NCTC $11864^{\mathrm{T}}$ & AGBA01 (ATCC $25577^{\mathrm{T} *}$ ) \\
\hline Cutibacterium granulosum & NCTC $11865^{\mathrm{T}}$ & LT906441 (NCTC 11865²) \\
\hline Cutibacterium modestum & JCM $33380^{\mathrm{T}}$ & BJEN01 $\left(\mathrm{M} 12^{\mathrm{T} *}\right)$ \\
\hline Cutibacterium namnetense & CCUG $66358^{\mathrm{T}}$ & LWHO01 (NTS $31307302^{\mathrm{T} *}$ ) \\
\hline Staphylococcus aureus & CCUG $1800^{\mathrm{T}}$ & CP011526 (DSM 20231 $\left.{ }^{\mathrm{T} *}\right)$ \\
\hline Staphylococcus capitis subsp. capitis & AYP 1020 & CP007601 (AYP 1020) \\
\hline $\begin{array}{l}\text { Staphylococcus capitis } \\
\text { subsp. urealyticus }\end{array}$ & CCUG $35142^{\mathrm{T}}$ & PPQI01 (DSM 6717 *) \\
\hline Staphylococcus caprae & DSM $20608^{\mathrm{T}}$ & PPRT01 (NCTC 12196 ${ }^{\mathrm{T}} *$ ) \\
\hline Staphylococcus epidermidis & $\mathrm{JCM} 2414^{\mathrm{T}}$ & CР035288 (ATCC $\left.14990^{\mathrm{T}} *\right)$ \\
\hline Staphylococcus hominis & JCM $31912^{\mathrm{T}}$ & PPQE01 (NCTC $\left.11320^{\mathrm{T} *}\right)$ \\
\hline Staphylococcus lugdunensis & CCUG $25348^{\mathrm{T}}$ & LS483482 (NCTC $\left.12217^{\mathrm{T} *}\right)$ \\
\hline
\end{tabular}

\subsection{Selection of Genome Data}

The whole-genome data for all strains were selected from the database. Some type strains had multiple entries under different strain names, caused by their deposition in multiple culture collections and resource swapping between collections. One of such examples is JCM $6425^{\mathrm{T}}$, the type strain of $C$. acnes and C. acnes subsp. acnes, listed in fourteen culture collections, according to the JCM online catalog (https://jcm.brc.riken.jp/en/, accessed on 5 June 2021). Its genome data was registered under the strain names of ATCC 6919 (accession numbers CP023676, CP044255, and JNHS01), DSM 1897 (CP025934 and AWZZ01), and NBRC 107605 (AP019723) as of March 2021. In the case of multiple genome submissions under different strain names for one strain, the genome data with the highest status and the latest registration date was selected for analysis (Table 1).

\subsection{MALDI-TOF Mass Spectrometry}

MALDI-TOF mass spectrometry profiles of the strains were obtained using a MALDI Microflex Biotyper (Bruker, Billerica, MA, USA) with parameters as follows: mass range 1960-20,137; ion source 1: $20 \mathrm{kV}$, 2: $18 \mathrm{kV}$; lens: $6 \mathrm{kV}$; detector gain voltage: $2500 \mathrm{~V}$ (linear base). For each strain, a cultured colony on the agar plate was taken by a wooden toothpick and smeared onto a target plate (MSP 96 target polished steel BC; Bruker) and fixed with $1 \mu \mathrm{L}$ HCCA ( $\alpha$-cyano-4-hydroxycinnamic acid) (Bruker). Two hundred laser shots were shot at one point of the target to acquire an accumulated spectrum of 200 spectra, and if the peak height reached a preset threshold, the spectrum was retained. The point in the target was randomly moved. When six accumulated spectra were obtained, all six were added and finalized. The spectra were obtained in triplicate at least, and the 
representative spectrum with the clearest and rich pattern was selected. The raw spectral data were analyzed using flexAnalysis v3.4 software (Bruker). The spectra data were submitted to jPOST Repository under accession number JPST001137 (PXID: PXD025627) (https:/ / repository.jpostdb.org/entry/JPST001137, accessed on 7 June 2021).

\subsection{Protein Profiling of 3-22 kD Range to Identify MALDI Peaks}

Five Cutibacterium strains (C. acnes subsp. acnes JCM $6425^{\mathrm{T}}$, C. acnes subsp. acnes JCM 18918, C. acnes subsp. defendens JCM 6473 ${ }^{\mathrm{T}}$, C. acnes subsp. elongatum JCM 18919 ${ }^{\mathrm{T}}$, and C. modestum JCM 33380 ${ }^{\mathrm{T}}$ ) were used. A loopful of bacterial cells for each strain was diffused to $2 \mathrm{~mL}$ distilled water and lysed by $5 \mathrm{~min}$ sonication using an Insonator 201M device (Kubota, Japan). The samples were concentrated under vacuum overnight and then diluted in distilled water to create $40 \mu \mathrm{L}$ of samples before preservation at $-80{ }^{\circ} \mathrm{C}$.

In-gel protein digestion was performed based on the methods of Shevchenko et al. [11] and Misra et al. [12]. First, $10 \mu \mathrm{g}$ protein samples in $20 \mu \mathrm{L}$ distilled water were prepared. After being placed in a $70{ }^{\circ} \mathrm{C}$ bath for $10 \mathrm{~min}$, the samples and PageRuler Low Range Unstained Protein Ladder (Thermo Fisher Scientific, Waltham, MA, USA) were run on NuPAGE 12\% Bis-Tris Gel (1.0 mm, 12 wells) (Thermo Fisher Scientific) with NuPAGE MES SDS Running Buffer (Thermo Fisher Scientific) $200 \mathrm{~V}$ for $35 \mathrm{~min}$. Coomassie blue staining was performed using SimplyBlue Safe Stain (Thermo Fisher Scientific), and bands comprising 3-5, 5-12, 12-15, and 15-22 kD were excised by a scalpel (Figure 1). The protein in the gel fragments was destained with $500 \mu \mathrm{L}$ of $10 \%$ acetic acid for $30 \mathrm{~min}$ and then washed with distilled water three times. Then, $500 \mu \mathrm{L}$ of $100 \%$ acetonitrile was added and placed at room temperature for $10 \mathrm{~min}$ before decantation and $5 \mathrm{~min}$ of drying. Reduction: 30-50 $\mu \mathrm{L}$ of $10 \mathrm{mM}$ DTT in $100 \mathrm{mM}$ ammonium bicarbonate was added and incubated at $56^{\circ} \mathrm{C}$ for $30 \mathrm{~min} ; 500 \mu \mathrm{L}$ of $100 \%$ acetonitrile was added and placed under room temperature for $10 \mathrm{~min}$ before decantation. The DTT amount was determined so that the liquid could cover the gel fragment. Alkylation: $30-50 \mu \mathrm{L}$ of $55 \mathrm{mM}$ iodoacetamide in $100 \mathrm{mM}$ ammonium bicarbonate was added and placed in the shade for $20 \mathrm{~min} ; 500 \mu \mathrm{L}$ of $100 \%$ acetonitrile was added and placed at room temperature for $10 \mathrm{~min}$ before decantation. Digestion: 50-100 $\mu \mathrm{L}$ of $10 \mathrm{mg} / \mathrm{mL}$ Trypsin Gold (Promega, Madison, WI, USA) in $100 \mathrm{mM}$ ammonium bicarbonate was added and incubated at $4{ }^{\circ} \mathrm{C}$ for $120 \mathrm{~min} ; 10-20 \mu \mathrm{L}$ of $100 \mathrm{mM}$ ammonium bicarbonate was added so that the gel piece was not exposed to air, and the sample was placed at room temperature overnight. The liquid around the gel was withdrawn and preserved at $-80^{\circ} \mathrm{C} ; 100 \mu \mathrm{L}$ of extraction buffer $(1: 2(\mathrm{vol} / \mathrm{vol}) 5 \%$ formic acid/acetonitrile) was added to each tube and shaken for $1 \mathrm{hr} ; 10-20 \mu \mathrm{L}$ of TFA was added before vortexing and centrifuging at $15,000 \times g$ for $20 \mathrm{~min}$. The aliquot was withdrawn and preserved at $-80{ }^{\circ} \mathrm{C}$.

The samples were then analyzed with the Synapt G2-Si UPLC-MS/MS system (Waters, Milford, MA, USA); $4 \mathrm{uL}$ of the digested materials were analyzed under $\mathrm{MS}^{\mathrm{E}}$ mode. The raw spectrum data were transferred to ProteinLynx Global Server v3.0.2 software (Waters) for peptide detection and protein identification using the Cutibacterium protein database, extracted from the UniProt public database with keywords "Cutibacterium", "sus scrofa trypsin", "yeast enolase" (yeast enolase was used for the detection check), and "human keratin".

\section{5. $\mathrm{m} / \mathrm{z}$ Value Estimation for Prominent Cutibacterium Peaks}

The experimental flow is outlined in Figure 2. The proteins with the masses close to $m / z$ values of the prominent MALDI peaks of C. acnes JCM $6425^{\mathrm{T}}\left(=\mathrm{ATCC} 6919^{\mathrm{T}}\right.$ ) were selected from the protein lists and labeled "candidate proteins". The amino acid sequences of "candidate proteins" in C. acnes JCM $6425^{\mathrm{T}}$ were extracted from the SwissProt database using the keyword search on the UniProt webpage (https: / / www.uniprot.org, accessed on 5 June 2021). The gene positions and the translated amino acid sequences of these "candidate proteins" in the three Cutibacterium type strain genomes (accession numbers CP044255 for C. acnes subsp. acnes type strain ATCC 6919 ${ }^{\mathrm{T}}$, CP003084 for C. ac- 
nes subsp. defendens type strain ATCC $11828^{\mathrm{T}}$, and LT906441 for C. granulosum type strain NCTC $11865^{\mathrm{T}}$ ) were searched using the NCBI tblastn webtool (https:/ /blast.ncbi. nlm.nih.gov/Blast.cgi, accessed on 5 June 2021). Then, by using the NCBI Sequence Viewer (https:/ / www.ncbi.nlm.nih.gov / projects/sviewer/, accessed on 5 June 2021), the amino acid sequences were checked and curated using the correspondent genome sequences as templates. The masses of these proteins were calculated by using the Protein/Peptide Editor function of MassLynx v4.2 software (Waters) and converted to $m / z$ values for singly charged ions $[\mathrm{M}+\mathrm{H}]^{+}$and doubly charged ions $[\mathrm{M}+2 \mathrm{H}]^{+}$using the equations below to check if these values matched the observed MALDI peaks (error range of 500 ppm was allowed).

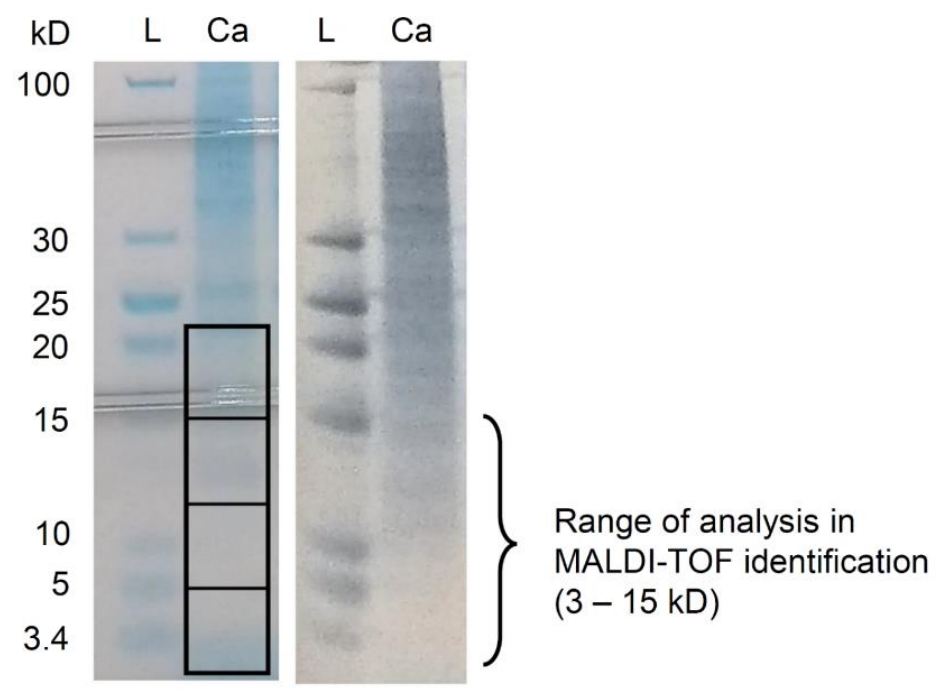

Figure 1. Example of $1 \mathrm{D}$ gel excision for proteomics. The material was extracted protein from Cutibacterium acnes subsp. acnes JCM 6425 $5^{\mathrm{T}}$. Left: Coomasie blue staining with SimplyBlue Safe Stain (Thermo Fisher Scientific). Rectangles indicate excision areas (3-5, 5-12, 12-15, and 15-22 kD). Right: reverse staining with ExStain Reverse (Atto, Tokyo, Japan). L: ladder. Ca: Cutibacterium acnes.

The 'one-species-fits-all' identification protocol for Cutibacterium species

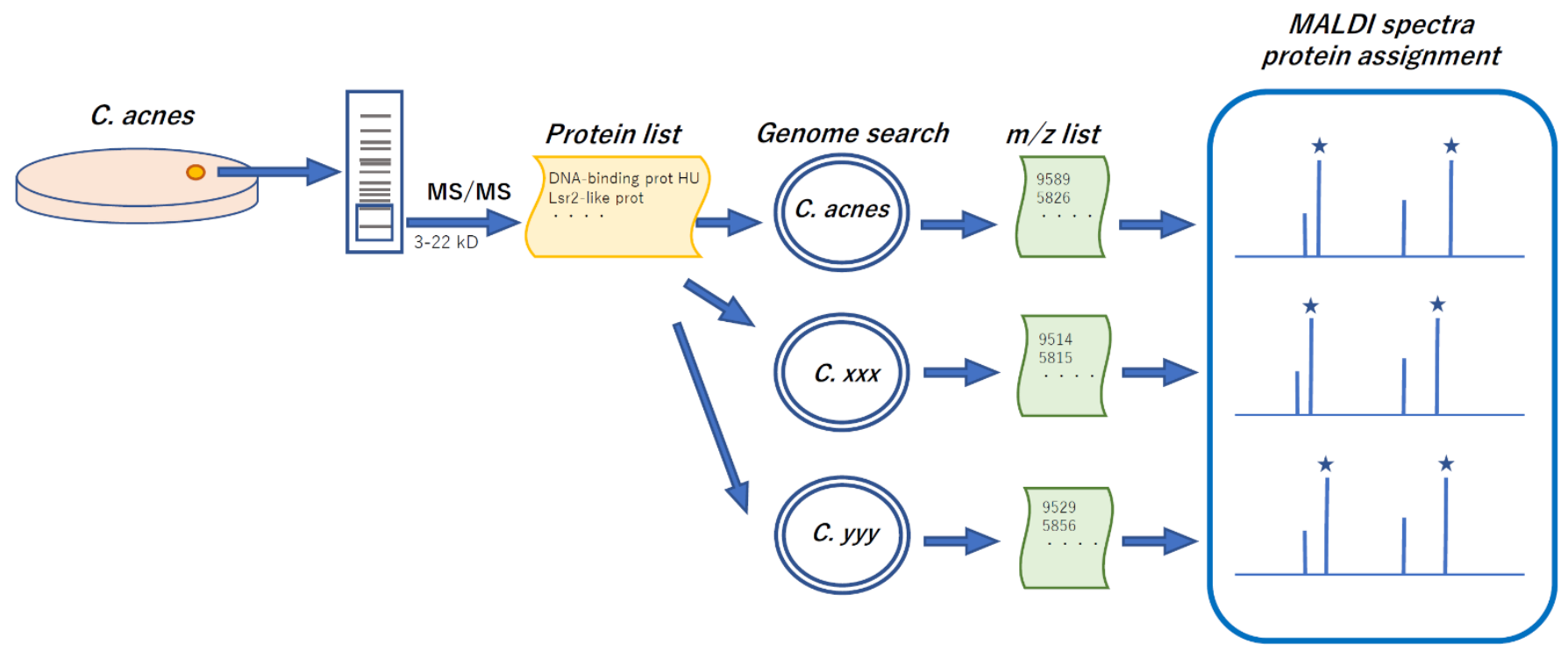

Figure 2. Scheme of the experimental flow for Cutibacterium MALDI peaks. 
For singly charged ions,

$$
m / z_{0}\left([\mathrm{M}+\mathrm{H}]^{+}\right)=(\text {molecular weight })+1.01
$$

For doubly charged ions,

$$
m / z_{0}\left([\mathrm{M}+2 \mathrm{H}]^{+}\right)=\{(\text {molecular weight })+1.01 \times 2\} / 2
$$

Additionally, the $m / z$ values of the amino acid sequences with $N$-terminal (posttranslational modification) Met or Val removed [3,13] were checked using the equations below based on the difference of the molecular weight without Met or Val, which were -130.49 and -98.12 , respectively.

For singly charged ions with N-terminal Met removed,

$$
m / z-\mathrm{M}\left([\mathrm{M}+\mathrm{H}]^{+}\right)=(\text {molecular weight }-130.49)+1.01
$$

For singly charged ions with N-terminal Val removed,

$$
m / z-\mathrm{v}\left([\mathrm{M}+\mathrm{H}]^{+}\right)=(\text {molecular weight }-98.12)+1.01
$$

For doubly charged ions with N-terminal Met removed,

$$
m / z-\mathrm{M}\left([\mathrm{M}+2 \mathrm{H}]^{+}\right)=\{(\text {molecular weight }-130.49)+1.01 \times 2\} / 2
$$

For doubly charged ions with N-terminal Val removed,

$$
m / z^{-\mathrm{v}}\left([\mathrm{M}+2 \mathrm{H}]^{+}\right)=\{(\text {molecular weight }-98.12)+1.01 \times 2\} / 2
$$

If either $m / z_{0}$ or $m / z_{-M} /-\mathrm{V}$ seemed to match a MALDI peak of all the three type strains of $C$. acnes subsp. acnes, C. acnes subsp. defendens, and C. granulosum, the same workflow was performed on the other type strain draft genomes (whole-genome shotgun project entries: BAVO for C. acnes subsp. acnes JCM 18918, BFFM for C. acnes subsp. elongatum JCM $18919^{\mathrm{T}}$, AGBA for C. avidum ATCC $25577^{\mathrm{T}}$, BJEN for C. modestum JCM 33380 ${ }^{\mathrm{T}}$, and LWHO for $C$. namnetense NTS $31307302^{\mathrm{T}}$ ) to check if the $\mathrm{m} / \mathrm{z}$ values also matched MALDI peaks of these five type strains.

\section{6. $\mathrm{m} / z$ Value Estimation for Prominent Staphylococcus Peaks}

First, we referred to the assignment result of Cutibacterium (7 proteins for 13 peaks) to create a list of 'candidate proteins'. The UniProt search using keywords as the protein name and 'Staphylococcus' was performed, and the retrieved amino acid sequence was used as a template for the tblastn search to find a corresponding genome sequence of $S$. aureus DSM $20231^{\mathrm{T}}$ (accession number: CP011526). Then, the DNA sequence was used to curate the template amino acid sequence. The $m / z$ calculation was done, as in the above Cutibacterium section, to see if the $m / z_{0}$ or $m / z_{-\mathrm{M} /-\mathrm{V}}$ values of each 'candidate protein' matched the observed peaks in the Staphylococcus MALDI spectrum. However, this workflow could assign only four peaks derived from two proteins and left the majority of the peaks unassigned. Secondly, we used the 2D gel proteomics results by Becher et al. [14] (Figure S1 of this reference, 40 proteins in the $<20 \mathrm{kD}$ area) to create another list of 'candidate proteins', and the same workflow was applied. This workflow could assign only one peak derived from one protein. Thirdly, we created a list of ribosomal subunit proteins coded on the S10-spc-alpha operon (24 proteins) [3] to serve as another list of 'candidate proteins' and performed the same analysis. Again, this workflow could assign only one peak derived from one protein.

Finally, we searched the S. aureus DSM $20231^{\mathrm{T}}$ genome to find possible proteins to form the peaks. The complete genome of the $S$. aureus subsp. aureus type strain, DSM $20231^{\mathrm{T}}$ (accession number: CP011526), was used to construct a genome-wide protein molecular 
weight list. Briefly, the annotated amino acid sequences were extracted, converted to a MultiFasta format, and then subjected to molecular weight calculation using online tools provided by the Belgian Co-ordinated Collections of Micro-organisms (https: / Www. genecorner.ugent.be/protein_mw.html, accessed on 7 June 2021) and the Swiss Institute of Bioinformatics (https:/ / web.expasy.org/compute_pi/, accessed on 7 June 2021). To visualize the molecular weight distribution, the number of proteins in each $\mathrm{kD}$ range was counted. The $\mathrm{N}$-end rule and the addition of a proton were applied to calculate $\mathrm{m} / z$. Similarly, we also constructed protein molecular weight lists for AYP 1020, DSM $6717^{\mathrm{T}}$, NCTC $12196^{\mathrm{T}}$, ATCC $14990^{\mathrm{T}}$, NCTC $11320^{\mathrm{T}}$, and NCTC $12217^{\mathrm{T}}$ using the complete genomes or sets of whole-genome shotgun scaffolds (accession Nos: CP007601, PPQI01, PPRT01, CP035288, PPQE01, and LS483482, respectively). An alignment of the $5.5 \mathrm{kD}$ hypothetical protein encoded in the genome of DSM $20231^{\mathrm{T}}, \mathrm{DSM} 6717^{\mathrm{T}}, \mathrm{NCTC}^{12196^{\mathrm{T}}}$, and NCTC $11320^{\mathrm{T}}$ was created using ClustalW 2.0 (GenomeNet, https:/ / www.genome.jp/ tools-bin/ clustalw, accessed on 7 June 2021). This workflow resulted in five peaks derived from five proteins.

\section{Results}

\subsection{MALDI-TOF Peak Comparison of Cutibacterium acnes across Different Oxygen Pressures}

Three Cutibacterium acnes strains were cultured under five different oxygen pressures. The MALDI-TOF peaks of the $7 \mathrm{kD}$ antitoxin and the CsbD-like protein were observed in all spectra, and the $7 \mathrm{kD}$ antitoxin peak height to the $\mathrm{CsbD}$-like protein peak height ratio was lower in lower oxygen pressures (Figure 3A).

\subsection{MALDI-TOF Mass Spectrometry Profiles of Genus Cutibacterium}

The spectra of the eight strains were successfully obtained. The spectra contained 13 prominent peaks, and the $m / z$ values of the MALDI-TOF peaks were different between strains.

On the other hand, 43 proteins were identified from the excised 1D gels in the 3-22 kD range of Cutibacterium extracts. These included 40 nonribosomal proteins and 3 ribosomal subunits. Among the nonribosomal proteins, 25 were with molecular weights less than $16 \mathrm{kD}$ (Table S1) and, thus, considered a pool of potential "candidate proteins". By calculating the masses of the protein ions listed in the UniProt database and those without an N-terminus amino acid of methionine or valine (see the example in Figure 3B), the singly charged ions of 6 proteins matched the peaks of multiple strains: Lsr2-like protein -Met $[\mathrm{M}+\mathrm{H}]^{+}$at $m / z 11,651.76$ for $C$. acnes subsp. acnes type strain, $10 \mathrm{kD}$ chaperonin GroS-Val $[\mathrm{M}+\mathrm{H}]^{+}$at $m / z$ 10,497.97, DNA-binding protein $\mathrm{HU}[\mathrm{M}+\mathrm{H}]^{+}$at $m / z$ 9589.07, CsbD-like protein -Val/-Met $[\mathrm{M}+\mathrm{H}]^{+}$at $m / z 7179.95,7 \mathrm{kD}$ antitoxin -Met $[\mathrm{M}+\mathrm{H}]^{+}$at $m / z$ 7034.57, and DUF3117 domain-containing protein -Met $[\mathrm{M}+\mathrm{H}]^{+}$at $m / z 5712.64$. The doubly charged ions of three out of these six proteins were also observed as MALDI peaks of multiple strains, which were Lsr2-like protein -Met $[\mathrm{M}+2 \mathrm{H}]^{2+}$ at $m / z 5826.39$ for C. acnes subsp. acnes type strain, $10 \mathrm{kD}$ chaperonin GroS -Val $[\mathrm{M}+2 \mathrm{H}]^{2+}$ at $m / z 5249.49$, and DNA-binding protein $\mathrm{HU}[\mathrm{M}+2 \mathrm{H}]^{2+}$ at $m / z$ 4795.04. Furthermore, using the analysis of genus Staphylococcus, as described later, other two peaks were assigned as the singly and doubly charged ions of $50 \mathrm{~S}$ ribosomal protein L7/L12, -Met $[\mathrm{M}+\mathrm{H}]^{+}$at $m / z$ 13,571.41 and -Met $[\mathrm{M}+2 \mathrm{H}]^{2+}$ at $m / z$ 6786.21, respectively (Figure 4, calculation in Tables S2 and S3).

These six proteins included two previously reported assignments, which were a CsbD-like protein and a $7 \mathrm{kD}$ antitoxin [3]. Our results were consistent with theirs except for the $\mathrm{m} / \mathrm{z}$ value for the $7 \mathrm{kD}$ antitoxin for C. acnes subsp. elongatum JCM $18919^{\mathrm{T}}$ at 6971.5 (calculated) or 6969.3 (observed) instead of 7004. 
A

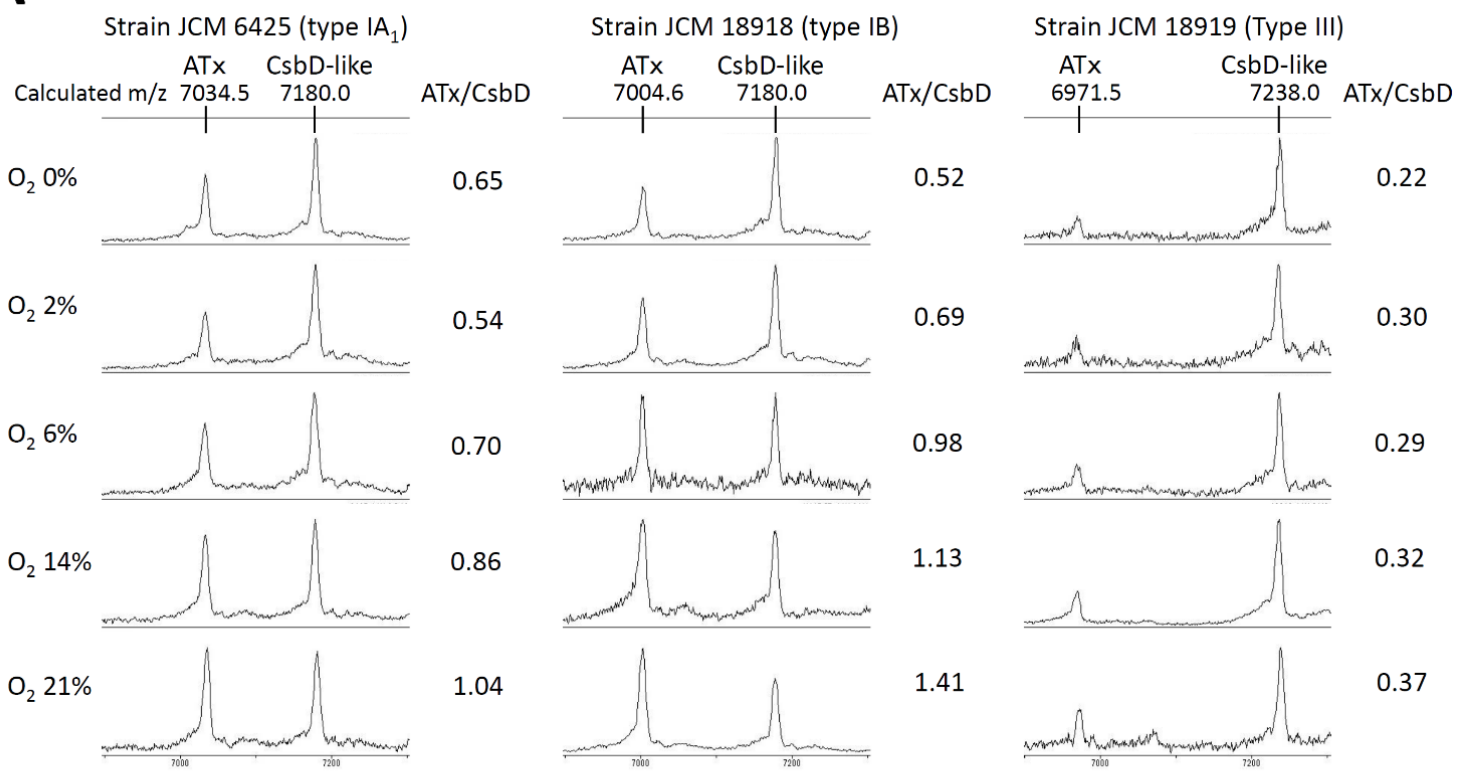

B Calculated amino acid sequences of $7 \mathrm{kD}$ antitoxin

C. acnes ssp. acnes Type IA Molecular weight $=7164.76, m / z_{0}=7165.77, m / z_{-M}=7034.57$

MGLFDKAKDAISDRQDDIKNQASQHSDQVEQGIDKAGNTVDDKTGGKFSDQIDKGQDALKDKLGDL

C. acnes ssp. acnes Type IB Molecular weight $=7134.73, m / z_{0}=7135.74, m / z_{-M}=7004.55$

MGLFDKAKDAISDRQDDIKNQASQHSDQVEQGIDKAGNAVVDDKGGKFSDQIDKGQDALKDKLGDL

C. acnes ssp. defendens (Type II) Molecular weight $=7115.68, m / z_{0}=7116.69, m / z_{-M}=6985.50$

MGLFDKAKDAISDHQDDIKNQASQHSDQVEQGIDKAGNAVDDKTGGKFSDQIDKGQDALKDKLGDL

C. acnes ssp. elongatum (Type III) Molecular weight $=7101.70, m / z_{0}=7101.71, m / z_{-M}=6971.52$

MGLFDKAKDA ISD $\underline{H} Q D D I K N Q A S Q H S D Q V E Q G I D K A G T A V \underline{N} D K T G G K E$ SDQIDKGQDALKDKLGDL

C. modestum Molecular weight $=7116.71, m / z_{0}=7117.72, m / z_{-M}=6986.53$

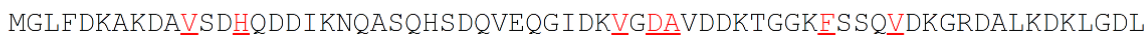

C. namnetense Molecular weight $=7070.65, \mathrm{~m} / \mathrm{z}_{0}=7071.66, \mathrm{~m} / \mathrm{z}_{\mathrm{M}}=6940.46$

MGLFDKAKDAINNHQDDIKNQASQHSDQVEQGIDKAGDAVDDKTGGKESSDQVDKGQDALKGKLGDL

C. avidum Molecular weight $=7173.80, \mathrm{~m} / \mathrm{z}_{0}=7174.81, \mathrm{~m} / \mathrm{z}_{-\mathrm{M}}=7043.62$

MGLFDKAKDAISD $\underline{H} Q D D I K \underline{D} Q \underline{V}$ SQHSDKVEQG IDKAGDIVDDKTGGKESSDQVDKGQDALKDKLGDL

C. granulosum Molecular weight $=7147.73, \mathrm{~m} / \mathrm{z}_{0}=7148.74, \mathrm{~m} / \mathrm{z}_{-\mathrm{M}}=7017.54$

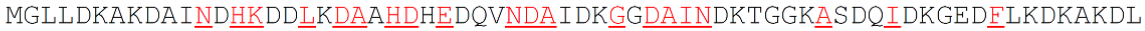

Figure 3. Background data for the MALDI peak assignment. (A) $7 \mathrm{kD}$ antitoxin and CsbD-like protein peaks of three Cutibacterium acnes strains cultured under different oxygen pressures. ATx: $7 \mathrm{kD}$ antitoxin, CsbD: CsbD-like protein, ATx/CsbD: peak height ratio of $7 \mathrm{kD}$ antitoxin and CsbD-like protein. Note that ATx/CsbD is higher when oxygen pressure is higher, which indicates the positive relationship between peak height and protein amount. (B) Comparison of amino acid sequences of $7 \mathrm{kD}$ antitoxin in Cutibacterium species and subspecies calculated from their genomes. Highlights with red and underlines indicate sequence differences from C. acnes subsp. acnes Type IA. The same calculations were also performed on the other 'candidate proteins'. 


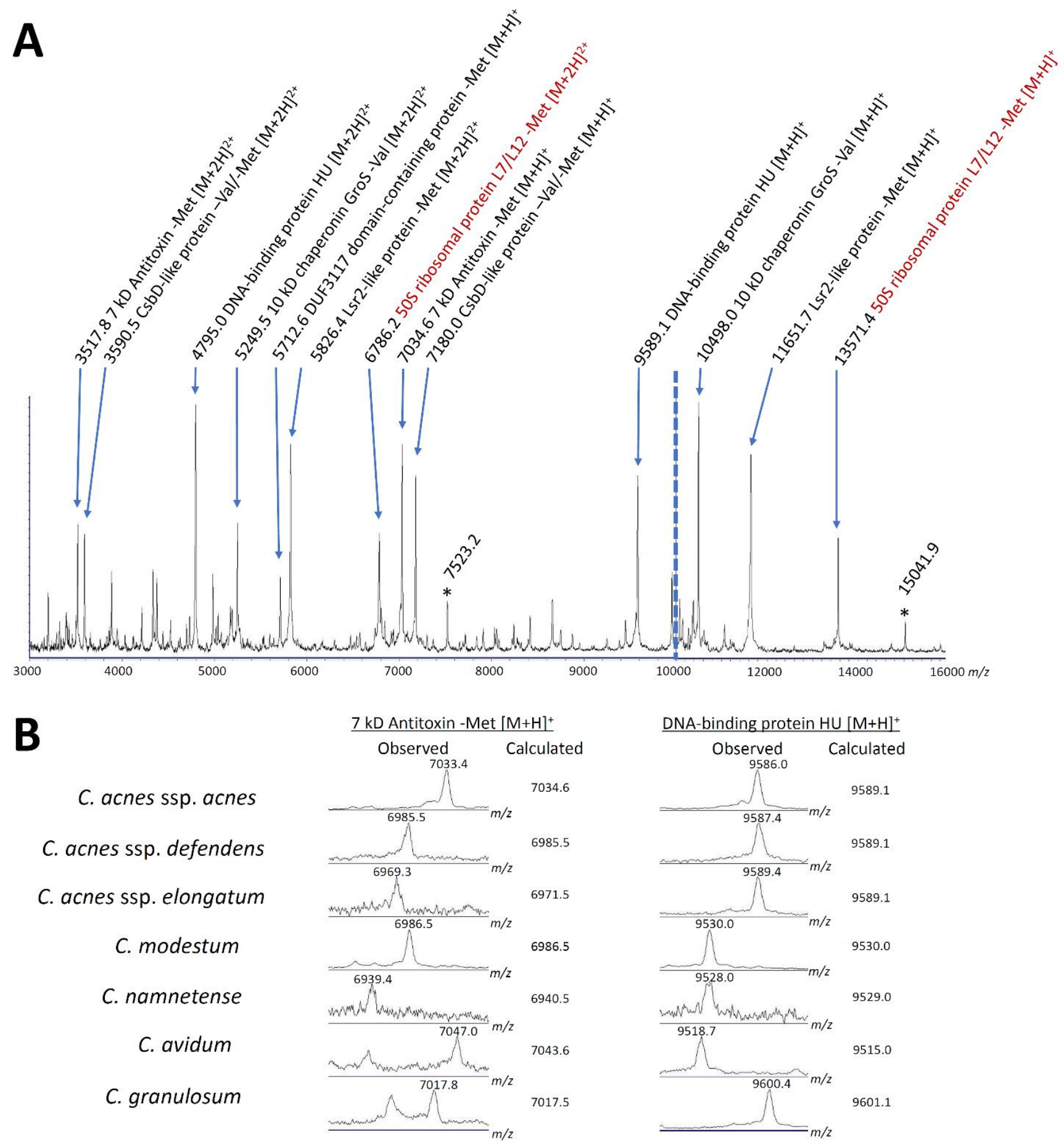

Figure 4. Assignment of prominent MALDI peaks of Cutibacterium acnes. (A) Peak assignment on the overall spectra of C. acnes subsp. acnes JCM $6425^{\mathrm{T}}$. Both $x$ - and $y$-axes are different between $3000-10,000 \mathrm{~m} / z$ and $10,000-16,000 \mathrm{~m} / \mathrm{z}$ ranges. Numbers before protein assignments are calculated $m / z$ values (observed $m / z$ values, see Table S2). Brown letters indicate ribosomal subunit proteins. -Met, -Val: methionine and valine at N-terminus deleted from original translations. * Nutrition-dependent peaks yet to be identified (singly and doubly charged ions of the same protein). (B) Examples of assignment by comparing the observed and calculated peaks. The calculation was performed by a bottom-up approach based on UPLC-MS/MS profiling of the proteins.

On the other hand, we could not characterize two peaks at 15,043 and 7522, even after adding the calculation considering the effects of disulfide binding, methylation, acetylation, and splicing. The $m / z$ values suggest these peaks are derived from the same 
protein: 15,043 being a singly charged ion and 7522 a doubly charged ion. These did not appear when we changed the agar plates from blood-containing plates (trypticase soy agar supplemented with $5 \%$ sheep blood, BD) to nonblood-containing plates (trypticase soy agar, BD), suggesting the peaks were derived from a blood component. Indeed, our UPLC-MS/MS analysis of Cutibacterium species using the whole protein list in the UniProt database resulted in a hemoglobin $\alpha$ chain, whose singly and doubly charged ions have calculated $m / z$ values close to these observed peaks (Table S3).

\subsection{MALDI-TOF Mass Spectrometry Profiles of Genus Staphylococcus}

The spectra of the seven strains were successfully obtained. Each spectrum contained 13 high-intensity peaks, and the $m / z$ values of these peaks were different between strains.

By using the result of genus Cutibacterium, four peaks were assigned for two proteins: DNA-binding protein $\mathrm{HU}\left([\mathrm{M}+\mathrm{H}]^{+}\right.$at $m / z 9627.02$ and $[\mathrm{M}+2 \mathrm{H}]^{2+}$ at $m / z 4814.02$ for $S$. aureus type strain) and CsbD-like protein $\left([\mathrm{M}+\mathrm{H}]^{+}\right.$at $m / z 6888.51$ and $[\mathrm{M}+2 \mathrm{H}]^{2+}$ at $m / z$ 3444.76). By using the protein list of a previous report by Becher et al. [14], one peak was assigned as a singly charged ion derived from a ribosomal subunit protein: $50 \mathrm{~S}$ ribosomal protein L7/L12 (-Met $[\mathrm{M}+\mathrm{H}]^{+}$at $\left.m / z 12,580.36\right)$. By using a list of ribosomal subunit proteins coded on the S10-spc-alpha operon, one peak was assigned as a singly charged ion derived from a ribosomal subunit protein: $50 \mathrm{~S}$ ribosomal protein $\mathrm{L} 36\left([\mathrm{M}+\mathrm{H}]^{+}\right.$at $\mathrm{m} / z$ 4306.37). Finally, to identify proteins corresponding to the remaining seven major peaks, we calculated the molecular weight and theoretical $\mathrm{m} / \mathrm{z}$ of whole coding sequences annotated in the complete genome of Staphylococcus aureus DSM 20231 ${ }^{\mathrm{T}}$ (see Materials and Methods). The amino acid sequence was available for 2606 coding sequences in the genome. Among these, the molecular weight of 523 proteins was within 3-15 kD, which is the detectable range of MALDI-TOF MS (Figure 5A). As a result of a comprehensive search using the genome-wide protein molecular weight list, we could assign five peaks as singly charged ions of four proteins and one putative protein: $30 \mathrm{~S}$ ribosomal protein S15 (-Met $[\mathrm{M}+\mathrm{H}]^{+}$at $m / z$ 10,478.07), $30 \mathrm{~S}$ ribosomal protein S16 (-Met $[\mathrm{M}+\mathrm{H}]^{+}$at $m / z$ 10,104.68), $50 \mathrm{~S}$ ribosomal protein L32 (-Met $[\mathrm{M}+\mathrm{H}]^{+}$at $m / z$ 6354.36), $5.5 \mathrm{kD}$ hypothetical protein (-Met $[\mathrm{M}+\mathrm{H}]^{+}$: 5525.00), and glycopeptide resistance-associated protein GraF (-Met $[\mathrm{M}+\mathrm{H}]^{+}$: 5032.49). These assignments consisted of a total of 11 out of 13 high-intensity peaks (Figure 5B,C; calculation in Tables S4 and S5).

On the other hand, two peaks at 15,043 and 7522 were observed in a similar pattern as genus Cutibacterium, and we could not identify these peaks. The $m / z$ values of these peaks seemed conserved across species. 

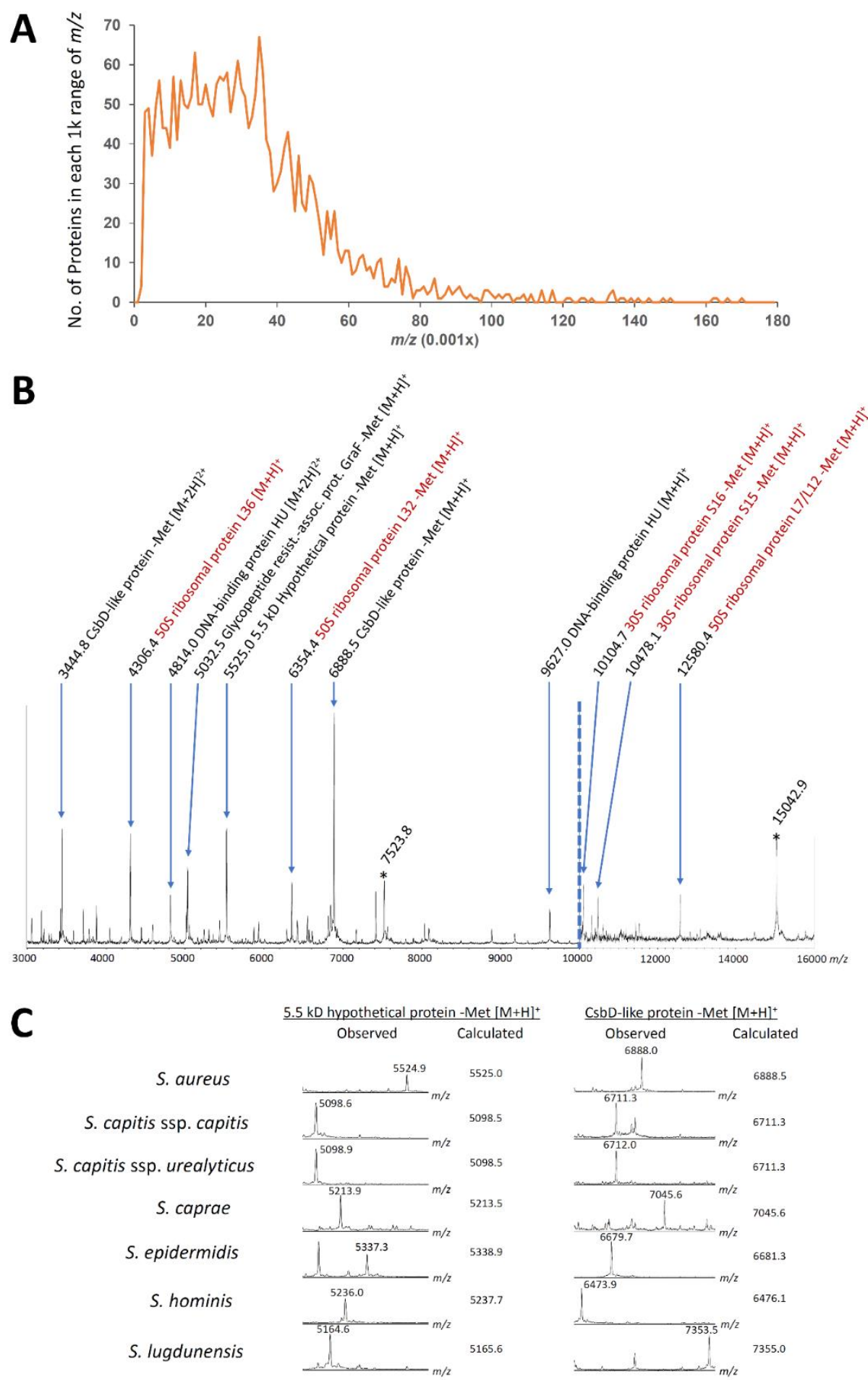

Figure 5. Assignment of prominent MALDI peaks of Staphylococcus aureus. (A) Number of proteins coded in the Staphylococcus aureus DSM 20231 ${ }^{\mathrm{T}}$ genome. Approximately 40-60 proteins are coded in each $1 \mathrm{kD}$ range. This indicates that the protein assignment can be confirmed when a calculated peak fits an observed peak within the 500 ppm range for multiple species. (B) Peak assignment on the overall spectra of S. aureus CCUG $1800^{\mathrm{T}}$. Both $x$-axis and $y$-axis are different between $3000-10,000 \mathrm{~m} / z$ and 10,000-16,000 $\mathrm{m} / z$ ranges. Numbers before protein assignments are calculated $\mathrm{m} / z$ values (observed $m / z$ values, see Table S4). Brown letters indicate ribosomal subunit proteins. -Met: methionine at $\mathrm{N}$-terminus deleted from original translations. ${ }^{*}$ Nutrition-dependent peaks yet to be identified (singly and doubly charged ions of the same protein). (C) Examples of assignment by comparing the observed and calculated peaks. The calculation was performed on Cutibacterium results, ribosomal subunit proteins coded on the S10-spc-alpha operon, and a 2D analysis report by Becher et al. [11]. 


\section{Discussion}

We could successfully assign the majority of the high-intensity peaks of MALDI-TOF spectra for Cutibacterium and Staphylococcus species by proteogenomic approaches, and this is the first report of such analysis for bacterial MALDI-TOF mass spectrometry. Our methodology includes a bottom-up approach by UPLC-MS/MS analysis, with gel excision and curation of amino acid sequences with genome data, and a top-down approach by protein-coding lesion analysis in a genome. In Cutibacterium spectra, only 2 out of 13 peaks were of ribosomal subunit proteins, making a striking contrast with Staphylococus spectra, in which 5 out of 11 peaks were of ribosomals. Our findings lead to a potential breakthrough for the development of a more precise and lighter database for MALDI-TOF microbial identification of bacterial species.

\subsection{Stability of MALDI Major Peaks and Rationale of Methodology}

We observed that the height ratio of the two major peaks around $7 \mathrm{kD}$ of MALDI-TOF spectra of Cutibacterium acnes showed a linear relationship with different oxygen pressures (Figure 3A). Although the mechanism causing this ratio difference remains unknown, this finding suggests that the peak heights are semiquantitative, and these major peaks should be similarly present in spectra for a strain under different laboratories.

During the assignment process, we noticed several proteins are present at a similar mass range, so that it is often confusing to characterize. The difference of the calculated masses across subspecies/species is helpful in determining the assignment. On the other hand, our genome analysis of the Staphylococcus aureus type strain showed that around 50 proteins were coded in a $1 \mathrm{kD}$ window of the $3-15 \mathrm{kD}$ area, where MALDI spectra are detected. As we applied a $500 \mathrm{ppm}$ error range, the difference between calculated and observed $m / z$ values was within 3.5 for $7 \mathrm{kD}$ and 5 for $10 \mathrm{kD}$. This means the top-down approach gives several proteins with $m / z$ values close to an observed peak. Therefore, a multispecies comparison within a genus is crucial for the final verification of the assignment.

\subsection{Identified Proteins Consisting of Major MALDI-TOF Spectra Peaks for Cutibacterium}

We identified six nonribosomal proteins, which were Lsr2-like protein, $10 \mathrm{kD}$ chaperonin GroS, DNA-binding protein HU, 7 kD antitoxin, CsbD-like protein, and DUF3117 domain-containing protein, forming the prominent peaks of Cutibacterium spectra. Among these six proteins, two (10 kD chaperonin GroS and DNA-binding protein $\mathrm{HU}$ ) were related to cell multiplication and one ( $7 \mathrm{kD}$ antitoxin) to immunity-like protection, while the functions of three (Lsr2-like protein, CsbD-like protein, and DUF3117 domain-containing protein) were unknown. The observation is that at least two of these proteins are related to replication; this may reflect the log phase when the bacterial cells were harvested; $7 \mathrm{kD}$ antitoxin and CsbD-like protein had been identified as consisting of the two useful MALDITOF peaks in discriminating C. acnes subspecies [15,16], which are at 7033 and 7179 for $C$. acnes subsp. acnes type $\mathrm{IA}_{1}[4]$.

Additionally, $10 \mathrm{kD}$ chaperonin GroS (GroES) is known to exist in most bacteria and creates a dimer with $60 \mathrm{kD}$ chaperonin GroL (GroEL) to work as a machine to fold other proteins [17]. DNA-binding protein HU is one of the histone-like DNA-binding proteins abundant in all prokaryotes, and it has the ability to stabilize double-stranded DNA against thermal denaturation [18]. The $7 \mathrm{kD}$ antitoxin is speculated as one of several toxin-antitoxin protection system members in Cutibacterium species. A UniProt database search with keywords antitoxin and Cutibacterium gave at least seven proteins, including HicA-HicB and VapC-VapB toxin-antitoxin pairs, which have been deeply investigated [19]. Unfortunately, the $7 \mathrm{kD}$ antitoxin is yet unnamed and uncoupled. Considering that the masses of these well-known pair proteins were of 7-14 kD in the database but did not form prominent peaks in MALDI spectra, this $7 \mathrm{kD}$ antitoxin is a more dominant member of the toxin-antitoxin system in Cutibacterium species that needs further research. Lsr2 is a protein that regulates gene expression in Mycobacterium tuberculosis [20]. To understand the 
role of the Lsr2-like protein we encountered, we checked the amino acid sequence of Lsr2 in Mycobacterium and compared this with the Lsr2-like protein of C. acnes. The Lsr2-like protein of C. acnes type strain DSM $1897^{\mathrm{T}}\left(=\mathrm{JCM} 6425^{\mathrm{T}}\right)$ (accession number A0A6B7EXG2) had only $44 \%$ similarity to Lsr2 in Mycobacterium tuberculosis type strain H37Rv. Due to the relatively low similarity score, we could not judge whether our Lsr2-like protein functions as Lsr2. In a similar search, the CsbD-like protein of C. acnes type strain JCM 18918 (accession number W4U2V9) had almost no similarity to CsbD (=ywmG) in Bacillus subtilis (accession number P70964), which is a stress response protein related to phosphate starvation [21]. This protein is studied only in B. subtilis, and so we have no information on how our CsbD-like protein works. DUF3117 is described in the Pfam protein database (as of Feb 2021) as a protein of unknown function restricted to phylum Actinobacteria, and we came to no explanation after an intensive search of the literature as well as of the DUF3117-domain-containing protein we encountered.

\subsection{Identified Proteins Consisting Major MALDI-TOF Spectra Peaks for Staphylococcus}

Many of the MALDI-TOF peaks of genus Staphylococcus were derived from ribosomal proteins ( 5 out of 13 peaks) and proteins described in the Cutibacterium section (4 out of 13 peaks; DNA-binding protein and CsbD-like protein). The other two proteins were a $5.5 \mathrm{kD}$ hypothetical protein and glycopeptide resistance-associated protein GraF. The former is a hypothetical small protein consisting of 48 amino acids, and we firstly described its existence from our comprehensive genome analysis. No similar protein has been reported so far, and, thus, no explanation can be made about its function at this stage. The latter is a small protein consisting of 44 amino acids, firstly reported in 2005 as one of the influential genes in decreasing glycopeptide susceptibility and cell wall thickness in S. aureus [22]; however, no other literature about this protein follows. Although not characterized further, the peaks corresponding to these two proteins are useful for the identification of bacteria by the MALDI-TOF method.

\subsection{Commonly Observed Peaks at $m / z 15,041 / 7522$ in Both Cutibacterium and Staphylococcus}

We observed two peaks at $m / z 15,041$ and 7522 in the spectra of the majority of the Cutibacterium and Staphylococcus strains, and we could not assign proteins to these in our analysis. Considering that the peaks uniformly occur at similar $m / z$ values across species, we speculated these do not derive from the bacterial strains. We used a trypticase soy agar plate supplemented with $5 \%$ sheep blood, and our speculation reached the culture methodology. When we changed the agar plate to a trypticase soy agar plate without blood, these peaks did not appear (Figure S2). Moreover, in the UPLC-MS/MS analysis of Cutibacterium, we frequently detected the hemoglobin $\alpha$ chain. Therefore, we speculate that these peaks are derived from an ingredient of the blood carried over from the agar plates, namely, hemoglobin $\alpha$ chain (calculated $m / z$ for sheep: -Met $[\mathrm{M}+\mathrm{H}]^{+} 15,034.18$ ), with the molecular weight of 15,040 (for singly charged $[\mathrm{M}+\mathrm{H}]^{+}, m / z=15,041$, for doubly charged $\left.[\mathrm{M}+2 \mathrm{H}]^{2+}, m / z=7522\right)$. However, we did not conclude the assignment for two reasons. First, we did not make a comparison of the peak to a standard. Second, $m / z$ values of the observed peaks were uniformly larger than calculated, about $10 \mathrm{~m} / z$, and we have no explanation for this discrepancy.

\subsection{Comparison of MALDI Spectra at Subspecies Level}

Several reports have stated that bacterial identification is possible down to subspecies level using MALDI-TOF mass spectrometry. For Cutibacterium species, Nagy et al. [15] and ourselves $[16,23]$ have reported that $C$. acnes subspecies can be identified by the $m / z$ values of two peaks around $7 \mathrm{kD}$; later, Teramoto et al. showed that these were a CsbD-like protein and a $7 \mathrm{kD}$ antitoxin [4]. Several other species that can be identified at the subspecies level from direct MALDI-TOF MS include Bifidobacterium animalis [24], Mycobacterium abscessus [25], and Fusobacterium nucleatum [26]. However, no such report can be found in Staphylococcus species. Our result shows no difference in $m / z$ values of major peaks is 
present in two subspecies of Staphylococcus capitis, and this indicates that these subspecies cannot be identified by direct MALDI-TOF MS. In this context, $C$. acnes and S. capitis subspecies showed a striking contrast, and a precise check of the protein-coding lesions of the genomes may reveal whether subspecies-level identifications can be achieved by MALDI-TOF fingerprinting.

\section{Conclusions}

While using MALDI-TOF MS for bacterial identification, the majority of the highintensity MS peaks were not identified at all in the fingerprint method; this leads to ambiguity and a large amount of labor for creating a database. The recently developed S10-GERMS method [3], which analyses the peaks corresponding to theoretical $\mathrm{m} / \mathrm{z}$ values calculated from parts of the genome, could make it possible to identify strains at species, subspecies, or even lower levels. This implies that MALDI-TOF MS bacterial identification, coupled with the bioinformatics approach, will be an important screening tool. Therefore, this study aimed to determine the nature of key high-intensity peaks ubiquitously observed in MALDI-TOF spectra based on assignment and validation by both top-down and bottomup procedures.

Since the S10-GERMS method represents the sequence variations of proteins (not only the house-keeping proteins but also the nonubiquitous proteins), the possibility of the S10-GERMS method combined with results from a genomic database will be developed as a highly reliable advanced method for phylogenetic analysis as well as bacterial discrimination at the strain level.

Our result that the high-intensity MS peaks are assignable, and the fact that a part of them are common between genera suggests that a part of high-intensity MS peaks can be calculated from their genomes in any bacterial species/subspecies, paving the way for the development of lighter and easier-to-use identification databases.

Supplementary Materials: The following are available online at https: / www.mdpi.com/article/ 10.3390 / microorganisms9061243/s1, Table S1. Amino acid sequences of the assigned proteins of Cutibacterium acnes subsp. acnes JCM $6425^{\mathrm{T}}$. Table S2. Comparison chart of observed prominent MALDI peaks and calculated masses of significant proteins in Cutibacterium spectra. Table S3. Amino acid sequences of the assigned proteins of Staphylococcus aureus CCUG $1800^{\mathrm{T}}$. Table S4. Comparison chart of observed prominent MALDI peaks and calculated masses of significant proteins in Staphylococcus spectra. Figure S1. Identification of ORFs encoding GraF in Staphylococcus strains. Figure S2. Comparison of MALDI-TOF spectra of Cutibacterium and Staphylococcus species growing on agar plates with and without sheep's blood.

Author Contributions: Conceptualization, I.D., Y.S., S.H.-T. and H.T.; Methodology, I.D., Y.S., S.H.-T. and H.T.; Software, Y.S. and H.T.; Validation, I.D., Y.S., S.H.-T. and H.T.; Formal Analysis, I.D., Y.S., S.H.-T. and H.T.; Investigation, I.D., Y.S., S.H.-T. and H.T.; Resources, I.D., Y.S., Y.M., H.T. and M.S.; Data Curation, I.D. and S.H.-T.; Writing-Original Draft Preparation, I.D. and S.H.-T.; WritingReview and Editing, I.D., Y.S., S.H.-T., H.T. and M.S.; Visualization, I.D., Y.S., S.H.-T. and H.T.; Supervision, Y.S., H.T. and M.S.; Project Administration, Y.S. and H.T.; Funding Acquisition, Y.S., Y.M., H.T. and M.S. All authors have read and agreed to the published version of the manuscript.

Funding: This work was supported by a Japan Society for the Promotion of Science (JSPS) Grant-inAid for Scientific Research (A) $20 \mathrm{H} 00415$ to H.T., S.H.-T. and I.D.

Institutional Review Board Statement: Not applicable.

Informed Consent Statement: Not applicable.

Data Availability Statement: The MALDI-TOF spectra data presented in this study are openly available in the jPOST Repository [https: / / repository.jpostdb.org/entry/JPST001137, accessed on 7 June 2021], reference number JPST001137 (PXID: PXD025627).

Acknowledgments: We thank Thanai Paxton of Nihon Waters K. K., Tokyo, Japan, and Raju Misra at the Natural History Museum, London, UK, for technical assistance and Anton Y. Peleg at Monash 
University, Melbourne, Australia, for kindly sharing Staphylococcus capitis subsp. capitis strain AYP 1020.

Conflicts of Interest: The authors declare that there is no conflict of interest.

\section{References}

1. Złoch, M.; Rodzik, A.; Pauter, K.; Szultka-Młyńska, M.; Rogowska, A.; Kupczyk, W.; Pomastowski, P.; Buszewski, B. Problems with identifying and distinguishing salivary streptococci: A multi-instrumental approach. Future Microbiol. 2020, 15, 1157-1171. [CrossRef]

2. Kostrzewa, M.; Maier, T. Criteria for development of MALDI-TOF mass spectral database. In MALDI-TOF and Tandem MS for Clinical Microbiology; Shah, H.N., Gharbia, S.E., Eds.; Wiley: West Sussex, UK, 2017; pp. 39-54, ISBN 978-1-118-96025-7.

3. Tamura, H. MALDI-TOF-MS based on ribosomal protein coding in S10-spc-alpha operons for proteotyping. In MALDI-TOF and Tandem MS for Clinical Microbiology; Shah, H.N., Gharbia, S.E., Eds.; Wiley: West Sussex, UK, 2017; pp. 269-310, ISBN 978-1-118-96025-7.

4. Teramoto, K.; Okubo, T.; Yamada, Y.; Sekiya, S.; Iwamoto, S.; Tanaka, K. Classification of Cutibacterium acnes at phylotype level by MALDI-MS proteotyping. Proc. JPN Acad. 2019, 95, 612-623. [CrossRef]

5. Brown, R.S.; Carr, B.L.; Lennon, J.J. Factors that influence the observed fast fragmentation of peptides in matrix-assisted laser desorption. J. Am. Soc. Mass Spectrom. 1996, 7, 225-232. [CrossRef]

6. Fagerquist, C.K.; Sultan, O.; Carter, M.Q. Possible evidence of amide bond formation between sinapinic acid and lysine-containing bacterial proteins by matrix-assisted laser desorption/ionization (MALDI) at $355 \mathrm{~nm}$. J. Am. Soc. Mass Spectrom. 2012, 23, 21022114. [CrossRef]

7. LPSN-List of Prokaryotic names with Standing in Nomenclature. Available online: https://lpsn.dsmz.de/ (accessed on 17 March 2021).

8. Scholz, C.F.; Kilian, M. The natural history of cutaneous propionibacteria, and reclassification of selected species within the genus Propionibacterium to the proposed novel genera Acidipropionibacterium gen. nov., Cutibacterium gen. nov. and Pseudopropionibacterium gen. nov. Int. J. Syst. Evol. Microbiol. 2016, 66, 4422-4432. [CrossRef]

9. Negi, M.; Takemura, T.; Guzman, J.; Uchida, K.; Furukawa, A.; Suzuki, Y.; Iida, T.; Ishige, I.; Minami, J.; Yamada, T.; et al. Localization of Propionibacterium acnes in granulomas supports a possible etiologic link between sarcoidosis and the bacterium. Mod. Pathol. 2012, 25, 1284-1297. [CrossRef]

10. Alexeyev, O.A.; Marklund, I.; Shannon, B.; Golovleva, I.; Olsson, J.; Andersson, C.; Eriksson, I.; Cohen, R.; Elgh, F. Direct visualization of Propionibacterium acnes in prostate tissue by multicolor fluorescent in situ hybridization assay. J. Clin. Microbiol. 2007, 45, 3721-3728. [CrossRef] [PubMed]

11. Shevchenko, A.; Tomas, H.; Havliš, J.; Olsen, J.V.; Mann, M. In-gel digestion for mass spectrometric characterization of proteins and proteomes. Nat. Protoc. 2006, 1, 2856-2860. [CrossRef] [PubMed]

12. Misra, R.V.; Ahmod, N.Z.; Parker, R.; Fang, M.; Shah, H.; Gharbia, S. Developing an integrated proteo-genomic approach for the characterisation of biomarkers for the identification of Bacillus anthracis. J. Microbiol. Methods 2012, 88, 237-247. [CrossRef]

13. Belinky, F.; Rogozin, I.B.; Koonin, E.V. Selection on start codons in prokaryotes and potential compensatory nucleotide substitutions. Sci. Rep. 2017, 7, 12422. [CrossRef] [PubMed]

14. Becher, D.; Hempel, K.; Sievers, S.; Zühlke, D.; Pané-Farré, J.; Otto, A.; Fuchs, S.; Albrecht, D.; Bernhardt, J.; Engelmann, S.; et al A proteomic view of an important human pathogen-towards the quantification of the entire Staphylococcus aureus proteome. PLoS ONE 2009, 4, e8176. [CrossRef]

15. Nagy, E.; Urbán, E.; Becker, S.; Kostrzewa, M.; Vörös, A.; Hunyadkürti, J.; Nagy, I. MALDI-TOF MS fingerprinting facilitates rapid discrimination of phylotypes I.; II and III of Propionibacterium acnes. Anaerobe 2013, 20, 20-26. [CrossRef]

16. Dekio, I.; Culak, R.; Misra, R.; Gaulton, T.; Fang, M.; Sakamoto, M.; Ohkuma, M.; Oshima, K.; Hattori, M.; Klenk, H.-P.; et al. Dissecting the taxonomic heterogeneity within Propionibacterium acnes: Proposal for Propionibacterium acnes subsp. acnes subsp. nov. and Propionibacterium acnes subsp. elongatum subsp. Int. J. Syst. Evol. Microbiol. 2015, 65, 4776-4787. [PubMed]

17. Hayer-Hartl, M.; Bracher, A.; Hartl, F.U. The GroEL-GroES chaperonin machine: A nano-cage for protein folding. Trends Biochem. Sci. 2016, 41, 62-76. [CrossRef] [PubMed]

18. Serban, D.; Arcineigas, S.F.; Vorgias, C.E.; Thomas, G.A., Jr. Structure and dynamics of the DNA-binding protein HU of B. stearothermophilus investigated by Raman and ultraviolet-resonance Raman spectroscopy. Protein. Sci. 2003, 12, 861-870. [CrossRef] [PubMed]

19. Unterholzner, S.J.; Poppenberger, B.; Rozhon, W. Toxin-antitoxin systems: Biology, identification, and application. Mob. Genet. Elem. 2013, 3, e26219. [CrossRef] [PubMed]

20. Summers, E.L.; Meindl, K.; Usón, I.; Mitra, A.K.; Radjainia, M.; Colangeli, R.; Alland, D.; Arcus, V.L. The structure of the oligomerization domain of Lsr2 from Mycobacterium tuberculosis reveals a mechanism for chromosome organization and protection. PLOS ONE 2012, 7, e38542. [CrossRef]

21. Prágai, Z.; Harwood, C.R. Regulatory interactions between the Pho and $\sigma^{\mathrm{B}}$-dependent general stress regulons of Bacillus subtilis. Microbiology 2002, 148, 1593-1602. [CrossRef]

22. Cui, L.; Lian, J.-Q.; Neoh, H.-M.; Reyes, E.; Hiramatsu, K. DNA microarray-based identification of genes associated with glycopeptide resistance in Staphylococcus Aureus. Antimicrob. Agents Chemother. 2005, 49, 3404-3413. [CrossRef] 
23. Dekio, I.; McDowell, A.; Sakamoto, M.; Tomida, S.; Ohkuma, M. Proposal of new combination, Cutibacterium acnes subsp. elongatum comb. nov., and emended descriptions of genus Cutibacterium, Cutibacterium acnes subsp. acnes, and Cutibacterium acnes subsp. defendens. Int. J. Syst. Evol. Microbiol. 2019, 69, 1087-1092.

24. Ruiz-Moyano, S.; Tao, N.; Underwood, M.A.; Mills, D.A. Rapid discrimination of Bifidobacterium animalis subspecies by matrixassisted laser desorption ionization time of flight (MALDI-TOF) mass spectrometry. Food Microbiol. 2012, 30, 432-437. [CrossRef] [PubMed]

25. Fangous, M.-S.; Mougari, F.; Gouriou, S.; Calvez, E.; Raskine, L.; Cambau, E.; Payan, C.; Héry-Arnaud, G. Classification algorithm for subspecies identification within the Mycobacterium abscessus species, based on matrix-assisted laser desorption ionization-time of flight mass spectrometry. J. Clin. Microbiol. 2014, 52, 3362-3369. [CrossRef] [PubMed]

26. Nie, S.; Tian, B.; Wang, X.; Pincus, D.H.; Welker, M.; Gilhuley, K.; Lu, X.; Han, Y.W.; Tang, Y.-W. Fusobacterium nucleatum subspecies identification by matrix-assisted laser desorption ionization-time of flight mass spectrometry. J. Clin. Microbiol. 2015, 53, 1399-1402. [CrossRef] [PubMed] 